\title{
Un modèle murin de maladie de Di George par recombinaison homologue d'un homéogène
}

Les gènes homéotiques ont été initialement définis chez la mouche par les conséquences de leurs mutations " homéotiques ", c'est-à-dire aboutissant à la transformation d'un segment du corps en un autre. Leurs produits sont caractérisés par la présence d'un domaine dit "homéo" qui a la structure "hélice-tourhélice " de nombreux facteurs transcriptionnels $\left(\mathrm{m} / \mathrm{s} n^{\circ} 7\right.$, vol. 3, p. 428). C'est principalement grâce à l'homologie entre les séquences nucléotidiques codant pour des homéodomaines chez la drosophile et les mammiferes qu'ont été isolés, chez ces derniers, les homéogènes qui sont les homologues du complexe homéotique antennapedia-ultrabithorax de la drosophile codant, comme eux, pour de très probables facteurs de transcription. Ces gènes sont disposés sur le chromosome dans l'ordre de leur expression sur l'axe antéropostérieur [1]. A certains stades du développement, les limites de leur territoire d'expression sont corrélées à l'organisation segmentaire du plan corporel : les segments de la mouche, les somites et, au niveau de la partie postérieure du cerveau, les rhombomères chez les vertébrés. Alors qu'il n'existe chez la drosophile qu'un seul locus antennapediaultrabithorax, quatre loci équivalents ont été détectés chez les mammifères, la souris et l'homme. Les gènes de ces loci sont désignés par l'abréviation hox, suivie du numéro du locus (1 à 4 ), puis de la position du gène dans ce locus. Le gène hox-1.5 est ainsi le cinquième gène hox du locus 1 . Il s'exprime très précocement au cours de l'embryogenèse de la souris, au niveau du cerveau postérieur, en avant des vésicules otiques. L'expression est maximale au niveau du neurectoderme, mais est également détectable au niveau du mésoderme avoisinant. Ultérieurement, des ARN messagers hox-1.5 sont détectés dans un plus grand nombre d'organes, le pharynx, le tronc aortique, la glande thyroïde, la moelle épinière, le poumon, l'estomac, la rate et les reins. Toutes ces données suggéraient fortement, mais ne prouvaient pas, que les gènes hox, tel hox-1.5, interviennent dans le contrôle du développement embryonnaire. Comme chez la drosophile où ce sont les mutations homéotiques qui ont permis de préciser la fonction de ces gènes, seuls des mutants peuvent permettre d'étudier directement l'influence des gènes hox sur le développement. La méthode de la recombinaison homologue permet d'inactiver un gène en introduisant à son niveau un fragment étranger d'ADN qui en interrompt la continuité ([2] et $\mathrm{m} / \mathrm{s} n^{\circ} 6$, vol. 6, p. 592). L'équipe de Mario R. Capecchi (Salt Lake City, UT, USA) vient ainsi de créer des embryons de souris homozygotes pour l'interruption du gène hox-1.5 par recombinaison homologue. Les souriceaux atteints meurent à la naissance, ou dans les heures qui suivent, du fait d'un syndrome malformatif complexe intéressant le cœur et les voies aériennes supérieures. De plus, ces souris ne possèdent pas de thymus, ni de glande parathyroïde, la glande thyroïde étant sousdéveloppée. Les malformations intéressent ainsi une partie des structures dans lesquelles le gène hox-1.5 est normalement exprimé au cours du développement, mais pas toutes. C'est ainsi que l'estomac, les poumons et les reins sont absolument normaux. Le syndrome malformatif des souris déficientes en la fonction hox-1.5 ressemble étrangement, par plusieurs aspects, à celui du syndrome de Di George chez l'homme (syndrome caractérisé par l'absence de thymus et de parathyroïde, par une hypoplasie thyroï- dienne ainsi que par des anomalies cranio-faciales et, parfois, cardiaques et artérielles). Cela ne signifie cependant pas que ce dernier syndrome soit imputable à une mutation du gène hox-1.5 humain. Le syndrome de Di George est, en effet, autosomique dominant, alors que la mutation du gène hox-1.5 est récessive (les animaux hétérozygotes sont strictement normaux). De plus, certains syndromes de Di George sont associés à des délétions et des translocations impliquant le chromosome 22, alors que le gène hox-1.5 humain est localisé sur le chromosome 7. Le gène du syndrome de Di George et le gène hox-1.5 pourraient, cependant, agir sur une séquence de développement commune. Cette séquence doit contrôler le destin de cellules appartenant à plusieurs lignages primitifs, neurectoderme et endoderme, et affecte des structures dérivées de plusieurs fentes et arcs brachiaux. Cette dernière observation peut d'ailleurs être secondaire au complexe mouvement de migration cellulaire impliqué dans la mise en place des structures de cette région. Quoi qu'il en soit, cette expérience est essentielle en ce qu'elle confirme sans aucune ambiguïté que les homéogènes de mammifères contrôlent, comme chez les invertébrés, la mise en place du plan corporel. Là s'arrête d'ailleurs, peut-être, la similitude. En effet, chez la drosophile, les pertes de fonction de gènes homéotiques aboutissent à la transformation de segments en segments plus antérieurs, alors que les mutations avec gain de fonction ont l'effet inverse, aboutissant à la transformation en segments plus postérieurs. Les résultats de l'inactivation du gène hox-1.5 ne sont pas l'apparition de structures d'un type plus antérieur. Au cours de l'évolution, il est probable que la complexification du schéma corporel, et donc du 
processus de développement, ait nécessité le recrutement d'un plus grand nombre d'homéogènes, ce qui aurait été obtenu par deux duplications successives des loci hox homologues du complexe antennapediaultrabithorax chez la drosophile. Les produits des gènes hox de ces différents loci pourraient finement contrôler le développement par une combinatoire d'interactions de telle sorte que le déséquilibre créé par l'inactivation d'un gène ait des résultats plus complexes que la seule transformation d'un segment en un autre. Cette duplication des gènes hox pourrait également expliquer l'absence d'anomalie d'autres tissus au niveau desquels s'exprime, néanmoins, le gène hox-1.5 au cours du développement. Il y aurait, dans ces cas, redondance fonctionnelle entre plusieurs gènes hox. C'est un tel phénomène de redondance fonctionnelle qui peut expliquer les résultats très singuliers de l'inactivation par recombinaison homologue de certains gènes dont le profil d'expression suggérait qu'ils étaient essentiels au développement. L'inactivation du gène src n'a, comme effet, qu'une ostéopétrose ( $\mathrm{m} / \mathrm{s} n^{\circ}$ 5, vol. 7, p. 509), alors que l'inactivation du gène pim-1 n'a aucune traduction détectable $(\mathrm{m} / \mathrm{s}$ $n^{\circ}$ 3, vol. 7, p. 290).

En conclusion, les homéogènes semblent avoir été utilisés tout au long de l'évolution pour contrôler le développement, mais le rôle qui leur est dévolu semble avoir évolué parallèlement à l'édification de structures corporelles de plus en plus complexes. Chez la drosophile, un rôle très précis, systématique, peut être attribué à beaucoup de gènes homéotiques, avec peu de compensations et de chevauchements. Par la suite, il semble que ces gènes soient devenus des élé- ments d'un langage dont la signification est créée par une combinaison d'éléments et non plus par un élément unique. Cette évolution expliquerait aussi bien la création de déséquilibres complexes que la possibilité de compensations fonctionnelles observées chez les mutants.

A.K.

1. Jacob F. L'unité du vivant. médecine/sciences $1990 ; 6: 122-7$.

2. Lemarchandel V, Montagutelli X. La recombinaison homologue : de nouvelles perspectives pour la transgenèse. médecine/sciences $1990 ; 6: 18-29$

3. Chisaka O, Capecchi MR. A genetically restricted developmental defects resulting from targeted destruction of the mouse homeobox gene hox-1.5. Nature $1991 ; 350: 473-9$.

\section{口曰 BRÈVES}

- Nanisme de souris transgéniques exprimant un mutant non phosphorylable de la protéine CREB dans les cellules somatotropes de l'hypophyse. La protéine CREB (cyclic AMP response elementbinding protein) est le principal intermédiaire des effets transcriptionnels de l'AMP cyclique. Cette protéine se fixe à l'ADN au niveau de courts motifs appelés CRE (cyclic AMP response element) ; elle est phosphorylable à la fois par la protéine kinase $\mathrm{C}$ et par la protéine kinase $\mathrm{A}$, cette dernière étant stimulée par l'AMP cyclique. Il semblerait que la phosphorylation par la protéine kinase $C$ controlât la dimérisation de $\mathrm{CREB}$, augmentant ainsi son affinité pour les éléments CRE. En revanche, la protéine kinase $\mathrm{A}$ induit la phosphorylation d'autres sites qui ne modifient pas l'affinité de la protéine pour les CRE, mais augmente son pouvoir d'activation transcriptionnelle. En l'absence de phosphorylation, CREB pourrait ainsi se fixer sur son élément CRE, mais être totalement incapable d'activer la transcription. Dans l'hypophyse, les cellules somatotropes répondent au GRF hypothalamique (growth hormone-releasing factor) par un mécanisme qui met en jeu l'activation de l'adénylate cyclase. Ex vivo, en culture de cellules, l'AMP cyclique a une action mitogène sur les cellules somatotropes. Afin de tester l'hypothèse selon laquelle le GRF activerait, au cours du développement, la prolifération des cellules somatotropes par un mécanisme impliquant la protéine CREB, R. S. Struthers et al., des laboratoires de Wylie Vale, et Marc Montminy (La Jolla, CA, USA) [1] viennent de créer des souris transgéniques exprimant un inhibiteur dominant de l'action physiologique de CREB. Pour ce faire, ils ont utilisé un ADN complémentaire codant pour CREB, muté au niveau de la Ser 133 en Ala ; cette Ser 133 est la cible de la phosphorylation catalysée par la protéine kinase A. L'ADNc muté a été mis sous le contrôle du promoteur de l'hormone de croissance. Les souris transgéniques exprimant cette construction CREB au niveau de leurs cellules somatotropes sont naines, avec une hypoplasie extrêmement importante des cellules somatotropes de l'hypophyse, alors que les autres populations cellulaires hypophysaires sont normales. Le mécanisme en est très probablement que la protéine CREB mutée se fixe sur les éléments CRE cibles, prenant la place de la protéine CREB normale ; contrairement à cette dernière, cependant, elle ne peut entraîner l'activation transcriptionnelle des gènes normalement contrôlés par l'AMP cyclique.

[1. Struthers RC, et al. Nature 1991 $350: 622-4$.

. 